\title{
Echocardiography in the Era of COVID-19: Lessons for the Future
}

\author{
Fitsumberhan Medhane ${ }^{1}$. James N. Kirkpatrick ${ }^{1}$
}

Accepted: 3 August 2021 / Published online: 16 October 2021

(c) The Author(s), under exclusive licence to Springer Science+Business Media, LLC, part of Springer Nature 2021

\begin{abstract}
Purpose of Review Poor cardiovascular outcomes are linked to COVID-19 in patients with or without prior cardiovascular disease or risk factors. Echocardiography, as a portable, versatile, and comprehensive imaging technique, has been on the frontlines. Yet sonographers and physician imagers are at increased risk of contracting or transmitting COVID-19.

Recent Findings Recent scientific statements incorporate triaging approaches to identify the appropriateness of imaging exam indications, coupled with triaging of indications. Additionally, focused protocols, procedures to reduce exposure, and point-of-care ultrasound play significant roles. Lessons learned during COVID-19 will apply to future pandemics.

Summary Echocardiography is a key diagnostic modality during pandemics in patients with or without prior cardiac diseases and risk factors. Attention to clinical questions, focused protocols, novel procedures, and future developments in imaging will contribute to safe and effective practice of echocardiography.
\end{abstract}

Keywords COVID-19 $\cdot$ Echocardiography $\cdot$ Pandemics

\section{Introduction}

Coronavirus disease (COVID-19) emerged as a major public health threat and was declared a pandemic early in its course. It has been responsible for significant mortality and morbidity, creating unprecedented pressure on healthcare services across the world $[1,2]$. It also has placed healthcare workers at risk because the virus can spread with close contact [3]. The virus has been shown to have higher morbidity and mortality in patients with established cardiovascular disease and cardiovascular risk factors, as well as the elderly, the immunocompromised, and the chronically ill.

Cardiovascular complications related to COVID 19 were noted early in the course of the pandemic. These include acute cardiac injury and inflammation, worsening of preexisting cardiac disease, acute right and left heart failure from primary cardiac pathology or due to pressure overload

This article is part of the Topical Collection on Echocardiography

James N. Kirkpatrick

kirkpatj@ cardiology.washington.edu

Fitsumberhan Medhane

fitsumb@cardiology.washington.edu

1 Division of Cardiology, University of Washington Medical Center, Seattle, WA 98195, USA secondary to lung disease, electrolyte and conduction disturbances, drug-related myocardial damage, and cardiovascular complications of hypercoagulable states. In addition, delayed presentation to medical care with cardiovascular signs and symptoms due to fears of contracting COVID 19 has contributed to significant morbidity and mortality.

Echocardiography is an inexpensive and widely accessible tool that can help provide important data to make important decisions in multiple clinical settings, including COVID-19 and, indeed, other pandemics. However, echocardiography is an intimate test, requiring close contact between the sonographer or physician performing the exam and the patient, for a significant period of time. Throughout the pandemic, frontline healthcare workers have been at a particular risk due to close contact with patients and increased chance of exposure to the virus, both from patients with established COVID-19 diagnoses and those with the virus who are asymptomatic or minimally symptomatic and are undiagnosed. In addition, echocardiography transducers and machines may serve as fomites. Performance of echocardiography during a pandemic therefore raises significant safety concerns. The value of performing echocardiography on specific patients with, or suspected of having, a highly contagious and dangerous disease and the use and versatility of echocardiography vis-à-vis other imaging modalities must be balanced with the importance of precautions to decrease risk of viral transmission and spread. 


\section{Echocardiography and Cardiac Findings in COVID-19}

The echocardiographic phenotype of patients with COVID-19 and its clinical significance has been an area of interest for clinicians taking care of these patients. Understanding cardiac manifestations of pandemic illness and characteristics of patients that would benefit from echocardiography is a crucial step toward judicious and appropriate use of heart ultrasound services.

During the early phases of the COVID 19 pandemic, a prospective international survey examined more than 1200 patients from 69 countries and six continents with presumed or confirmed COVID 19 who underwent echocardiography [4]. In this study, medical professionals who performed echocardiographic studies were surveyed after performance of the exams. Half of the patients involved (55\%) had abnormal echocardiogram findings. Of these, the most common findings include left and right ventricular abnormalities (39\% and 33\%, respectively), new myocardial infarction (3\%), myocarditis (2\%), and stress-induced cardiomyopathy (2\%). Severe cardiac disease, including severe ventricular dysfunction or tamponade, was observed in $15 \%$. More importantly, data from these echocardiography exams changed management in one-third of the patients, highlighting their importance in selected patient populations. Of note, this study did not fully account for established cardiovascular disease prior to COVID-19.

A retrospective observational cohort study described 74 patients with confirmed COVID-19 who had an indication for a transthoracic echocardiogram based on clinical parameters (chest pain, arrhythmia, abnormal electrogram changes, or hemodynamic instability) and a positive biomarker for myocardial injury[5]. It reported that $41 \%$ had right ventricular (RV) dilatation and $27 \%$ had impaired RV function. RV systolic dysfunction was significantly associated with pulmonary embolism (20\%). Its presence was associated with a prothrombotic, inflammatory state reflected in elevated d-dimer and CRP levels. A similar study found that in patients classified as having severe COVID-19, chamber enlargementspecifically, right ventricle, left ventricle, right atrium, and IVC diameters-was higher than in those without severe disease [6]. Pericardial effusions and reduced LV ejection fraction (LVEF) were also more frequent. Interestingly, significant correlations existed between RV diameter, LVEF, and elevated biomarkers (troponin I, CRP, and d-dimer levels).

Although there is much still to learn about the chronic effects of COVID-19, cardiac imaging, particularly echocardiography, will play an important role in defining lingering cardiac manifestations. It has already been recommended to assist in assessing the safety of return to athletic participation following infection [7].

\section{Cardiac Imaging in Pandemics}

\section{Appropriate Use Criteria}

Appropriate use criteria (AUC) serve as guides to the performance of cardiovascular imaging and therapeutic procedures. They consist of descriptions and rating of indications and clinical scenarios according to their level of appropriateness (rarely appropriate, may be appropriate, appropriate). The AUC are intended to guide clinicians in the ordering and performance of high value procedures. The Centers for Medicare and Medicaid Services (CMS) adopted AUC as part of its mandate for clinical decision support and will mandate the use of "clinical decision support mechanisms" at the time of test ordering for multiple services (https://www.cms.gov/ medicare/quality-initiatives-patient-assessment-instruments/ appropriate-use-criteria-program). Echocardiography is not yet included among these, but AUC for echocardiography have influenced pre-approval algorithms of healthcare insurance companies.

The AUC take on a new importance in the setting of a pandemic $[8 \bullet \bullet]$. Avoidance of low value imaging is important at all times in order to reduce unnecessary costs and prevent adverse downstream consequences that may arise from nontoxic but inappropriate tests, but pandemic conditions that put sonographers and physicians at risk and may serve to increase pathogen transmission elevate this importance. General principles underlying many of the AUC for echocardiography include avoidance of testing that (1) does not accompany a new or changed sign or symptom of cardiovascular disease, (2) is "routine," (3) will not change management (with some exceptions), and (4) constitutes screening. Essentially, there should be an adequate clinical justification for performing an echocardiogram during a pandemic, particularly on a patient with, or suspected of, having a highly transmissible and dangerous disease.

\section{Timing of Exam}

Appropriateness of indications is not the only important consideration. Echocardiography may yield important clinical information in a given scenario, but the "appropriate" timing of the exam may be impacted by pandemic conditions [9]. Many echocardiographic studies ordered for valid indications can be rescheduled for a later time in patients with, or suspected of having, a highly transmissible disease. Many patients whose disease status is unknown can wait for echocardiography until their status is known. In both of these situations, the inconvenience of delay is offset by the preservation of personal protective equipment and reduced sonographer/physician exposure. Depending on the situation during a pandemic, it is necessary to triage 
tests in one of two ways. The more lenient strategy consists of identifying patients and clinical scenarios in which an echocardiogram could be safely deferred and performing the rest. This method may be particularly appropriate when personal protective equipment and/or testing or vaccines are in adequate supply. It has the advantage of ensuring that patients who might benefit will receive the exam, essentially "doing the most good," but risks some sonographer and physician exposure. Alternatively, in a situation of short supply of personal protective equipment, limited testing capacity, no vaccines, and/or particularly high infectivity, it may be preferable to identify patients and clinical scenarios that absolutely require echocardiography (emergent or urgent cases), deferring all the rest. This more restrictive strategy theoretically "avoids the most harm" in terms of transmission but may end up delaying diagnoses to the detriment of patients. Furthermore, this strategy may contribute to taxing of healthcare resources because opportunities for earlier intervention to prevent disease progression are lost. In either case, the aim is to avoid morbidity and mortality in patients and healthcare workers, and concerns over transmission must be balanced by the benefits (and avoidance of harm) inherent in timely and accurate diagnosis.

\section{Exam Types and Locations}

Imaging modalities and test-performance factors also come into play. Echocardiography is one of the most versatile of all cardiac imaging modalities. It can be performed in multiple different settings; most importantly during a pandemic, all echocardiography procedures can take place in isolation rooms. Depending on staffing support and local protocols, transthoracic, transesophageal, and even stress echocardiography (dobutamine and other pharmacologic stress tests, including myocardial contrast imaging, and, sometimes, bicycle stress using portable cycle units) can take place without moving an infected patient through the hospital to an imaging lab, thereby reducing risk of transmission and saving personal protective equipment. Furthermore, cleaning of an echocardiogram machine is much easier than cleaning an entire room in an imaging lab.

Yet echocardiography puts sonographers and physician imagers in close proximity to patients for prolonged periods of time. According to the American Society of Echocardiography recommendations, an examination should be allocated "Forty-five to $60 \mathrm{~min}$ for the acquisition of the images for a complete TTE study. An additional 15 to 30 min may be required for complicated cases" [9]. Even a limited exam can take considerable time. Echocardiography for patients with highly transmissible infections should as brief as possible, with image acquisition focused on answering the clinical question. That said, it is sometimes appropriate to obtain additional imaging that may reduce the risk of transmission by making other tests unnecessary. For example, the performance of lung ultrasound during echocardiography may obviate the need for chest radiography or computed tomography. Reports and images from prior tests (cardiac ultrasound exams and other cardiac imaging modalities) should be reviewed in order to avoid unnecessary duplication. Simple practices may reduce exposure time, such as bringing ultrasound enhancing agents (UEAs) into the room of an infected patient, not only to reduce the amount of time required to wait for the agent but also to facilitate early use, rather than spending time trying to optimize endocardial definition without UEAs. Measurements should never be performed in the patient room but rather on the machine or workstation after

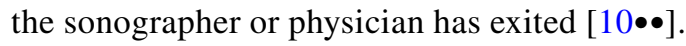

Additional measures to reduce the risk of transmission include "stripping down" ultrasound machines of anything but essentials, often including ECG leads (use time acquisition modes when possible) and extra transducers. Barriers between patients and sonographers should be considered [10••]. Similar considerations should be employed for perioperative TEEs, with machines wrapped in a protective layer [11•].

Risk of transmission of respiratory viruses is heighted during transesophageal echocardiography (TEE), during which patients cannot be masked and may cough, possibly leading to aerosolization of viral particles. TEEs should not be performed when transthoracic (TTE) imaging can more safely answer the clinical question in a patient with or suspected of having COVID 19, including in the perioperative setting [11•]. For example, TTE with agitated saline contrast ("bubble") injection can identify most significant interatrial shunts, and cardiac CT is demonstrating great promise in assessing the left atrial appendage for thrombus. Exercise stress tests, during which patients may breathe heavily or cough, may also raise the risk of transmission, especially as many patients will be unable to reach the target level of exercise while masked. Dobutamine stress testing and other modalities for assessing ischemia may provide adequate and safer (vis-à-vis COVID-19) alternatives for the detection of ischemia. Ideally, the infectious status of a patient should be known prior to TEE and exercise stress testing so that proper protective equipment can be employed $[8 \bullet \bullet$. Performance of imaging studies in ICU or operative settings where patients are receiving mechanical ventilation also raises risks, and echocardiography should be deferred, if possible, until patients are no longer ventilated.

\section{Value of POCUS}

Point-of-care ultrasound (POCUS) for cardiac studies in a focused setting can play a vital role in answering clinical questions and guiding management of cardiovascular 
disorders while minimizing exposure of healthcare workers. This becomes particularly important in settings where frontline essential workers also have the necessary skills to perform these studies. A prime example is critical care units. An early case series from Wuhan, China, described the use of point-of-care ultrasound, limited conventional transthoracic echocardiography, and critical care echocardiography in patients with COVID-19 to identify distinct clinical pathologies and change management [12]. In this case series, real-time images were obtained, and information derived from these was utilized to inform bedside decisions. For example, the use of point-of-care ultrasound identified the presence of unsuspected left ventricular dysfunction and valvular dysfunction. While identification of the former helped in clinical decision-making, further quantification of valvular dysfunction was not done given concerns about reducing image acquisition time and hence exposure. Several protocols that identify key components of POCUS imaging for COVID-19 have been published $[13,14]$. Technological advances have enabled several options for remote guidance of novice scanners. Some handheld ultrasound systems include software that allows a remote expert to see not only the image that the novice is creating but also the hand position. In the setting of a pandemic, this technology can increase the efficiency and effectiveness of scans and potentially reduce exposure. Newer devices have built-in artificial intelligence that guides novice scanners to produce diagnostic quality images and, in some cases, helps them to recognize what they are seeing [15]. Even though expert echocardiography with full feature devices will often be necessary, POCUS images can be used to direct and focus the subsequent echocardiogram exams, thus limiting exposure.

\section{Conclusions: Lessons Learned for Echocardiography in Future Pandemics} (Fig. 1)

It is highly likely that echocardiography will play a major role in future pandemics. The ability to rapidly assess cardiovascular anatomy and function and assess hemodynamics is essential in critical care settings, particularly the cardiac impact of systemic inflammation on patients with preexisting cardiovascular disease. Furthermore, pandemics have an important indirect impact by inducing delayed presentation of illnesses such as myocardial infarction and heart failure, and echocardiography will serve a triage function in identifying patients at elevated risk who will benefit from hospitalization and invasive interventions.

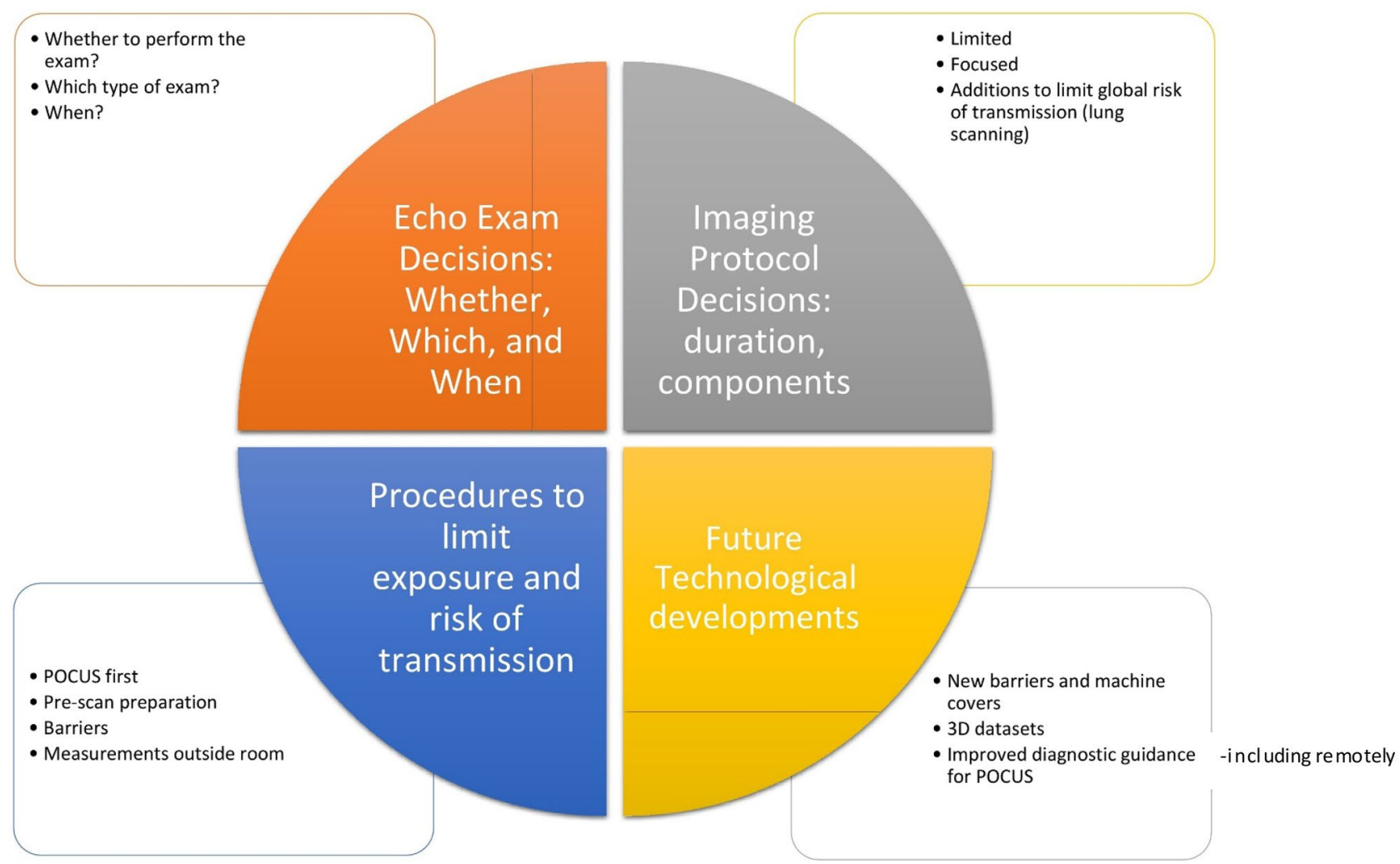

Fig. 1 Echocardiography during pandemics: triage, precautions, and future directions. POCUS, point-of-care ultrasound 
Table 1 Practical measures in echocardiography to reduce risks of transmission in a pandemic

\begin{tabular}{ll}
\hline Adherence to AUC & Avoid imaging that \\
1. Does not accompany a new or changed sign or symptom of cardiovascular disease \\
2. Is "routine" \\
3. Will not change management \\
4. (with some exceptions) constitutes screening \\
Defer exams when possible until infection or vaccination status is known General triage strategies \\
1. Lenient-identify exams that can be deferred and perform the rest \\
2. Restrictive-identify and perform only urgent/emergent exams \\
Triage and timing \\
Review prior reports, images, and other imaging studies to facilitate targeted imaging and avoid duplication \\
Bringing UEA into isolation rooms \\
"Strip down" machines \\
Barriers and machine covers \\
Focused imaging to answer a clinical question \\
Obtain additional images that prevent transmission risk (lung ultrasound) \\
Perform all measurements outside isolation room \\
Consider alternatives to TEE and exercise stress testing
\end{tabular}

UEA ultrasound enhancing agents

In future pandemics, it will again be necessary to protect frontline sonographers and physicians (and patients) by creating processes to determine whether echocardiogram exams should be performed, which imaging modalities should be used, and when these exams should be performed. Strict adherence to AUC is a cornerstone of this process. Rapid institution of measures to triage echocardiography orders should be instituted. Depending on the situation, it may be necessary either to defer all exams except those that are urgent or emergent (restrictive strategy), or focus on the identifying exams that can be safely deferred and perform the rest (lenient strategy), but recognize that the more lenient strategy may result in earlier identification of disease, allowing intervention at a stage that does not require hospitalization. TEEs and exercise stress testing that increase the risk of transmission should be subject to a more restrictive strategy of triage. Regardless, the duration of echo exams should be limited by focusing on images that answer clinical questions, but in some cases, additional imaging (e.g., lung imaging) may reduce global risks of exposure. Other simple practices can limit exposure (Table 1). In the future, rapid acquisition of wide-angle 3D datasets that can be cropped and measured outside of an insolation room may play a role in limiting scanning time. New barrier methods to reduce transmission between sonographer/physician and patients may become standard. The use of cardiopulmonary POCUS will increase, with less experienced scanners who enter isolation rooms as part of routine clinical care receiving guidance in acquisition of diagnostic images.

\section{Compliance with Ethical Standards}

Conflict of Interest The authors declare no competing interests.
Human and Animal Rights and Informed Consent This article does not contain any studies with human or animal subjects performed by any of the authors.

\section{References}

Papers of particular interest, published recently, have been highlighted as:

- Of importance

• Of major importance

1. Guan WJ, Ni ZY, Hu Y, Liang WH, Ou CQ, He JX, et al. Clinical characteristics of coronavirus disease 2019 in China. N Engl J Med. 2020;382:1708-20.

2. Gates B. Responding to Covid-19 - a once-in-a-century pandemic? N Engl J Med. 2020;382:1677-9.

3. Zhou F, Yu T, Du R, Fan G, Liu Y, Liu Z, et al. Clinical course and risk factors for mortality of adult inpatients with COVID-19 in Wuhan, China: a retrospective cohort study. Lancet. 2020;395:1054-62.

4. Dweck MR, Bularga A, Hahn RT, Bing R, Lee KK, Chapman $\mathrm{AR}$, et al. Global evaluation of echocardiography in patients with COVID-19. Eur Heart J Cardiovasc Imaging. 2020;21(9):94958. https://doi.org/10.1093/ehjci/jeaa178. PMID: 32556199; PMCID: PMC7337658.

5. Mahmoud-Elsayed HM, Moody WE, Bradlow WM, KhanKheil AM, Senior J, Hudsmith LE, et al. Echocardiographic findings in patients with COVID-19 pneumonia. Can J Cardiol. 2020;36(8):1203-1207. https://doi.org/10.1016/j.cjca.2020.05.030. Epub 2020 May 28. PMID: 32474111; PMCID: PMC7255734.

6. Barman HA, Atici A, Tekin EA, Baycan OF, Alici G, Meric $\mathrm{BK}$, et al. Echocardiographic features of patients with COVID19 infection: a cross-sectional study. Int J Cardiovasc Imaging. 2021;37(3):825-834. https://doi.org/10.1007/s10554-020-020519. Epub 2020 Oct 8. PMID: 33030636; PMCID: PMC7541759.

7. Phelan D, Kim JH, Chung EH. A game plan for the resumption of sport and exercise after coronavirus disease 2019 (COVID19) infection. JAMA Cardiol. 2020;5(10):1085-6. https://doi. org/10.1001/jamacardio.2020.2136 (PMID: 32402054). 
8.•• Kirkpatrick JN, Mitchell C, Taub C, Kort S, Hung J, Swaminathan M. ASE Statement on protection of patients and echocardiography service providers during the 2019 novel coronavirus outbreak: endorsed by the American College of Cardiology. J Am Soc Echocardiogr. 2020;33(6):648-653. This is the parent ASE guideline article that discusses the necessary precautions and approach to while performing echocardiography during COVID 19 pandemic.

9. Picard MH, Adams D, Bierig SM, Dent JM, Douglas PS, Gillam LD, et al. American Society of Echocardiography recommendations for quality echocardiography laboratory operations.; American Society of Echocardiography. J Am Soc Echocardiogr. 2011;24(1):1-10. https://doi.org/10.1016/j.echo.2010.11.006.

10.• Mitchell C, Collins K, Hua L, McClanahan C, Shea E, Umland $\mathrm{M}$, et al. Specific considerations for sonographers when performing echocardiography during the 2019 novel coronavirus outbreak: supplement to the American Society of Echocardiography Statement. J Am Soc Echocardiogr. 2020;33(6):654-657. https://doi.org/10.1016/j.echo.2020.04.014. Epub 2020 Apr 11. PMID: 32503701; PMCID: PMC7151492. Findings from this study depicts strategies that are helpful when performing echocardiography during COVID 19 and focuses on whom, where and how to scan.

11. Nicoara A, Maldonado Y, Kort S, Swaminathan M, Mackensen GB. Specific considerations for the protection of patients and echocardiography service providers when performing perioperative or periprocedural transesophageal echocardiography during the 2019 novel coronavirus outbreak: council on perioperative echocardiography supplement to the statement of the American Society of Echocardiography endorsed by the Society of Cardiovascular Anesthesiologists. J Am Soc Echocardiogr. 2020;33(6):666-669. https://doi.org/10.1016/j.echo.2020.04. 008. Epub 2020 Apr 11. PMID: 32503703. This study shows the necessary precautions that need to be taken during transesophageal echocardiography which carries high degree of airborne transmission.

12. Zhang L, Wang B, Zhou J, Kirkpatrick J, Xie M, Johri AM. Bedside focused cardiac ultrasound in COVID-19 from the Wuhan epicenter: the role of cardiac point-of-care ultrasound, limited transthoracic echocardiography, and critical care echocardiography. J Am Soc Echocardiogr. 2020;33(6):676-82.

13. Johri AM, Galen B, Kirkpatrick JN, Lanspa M, Mulvagh S, Thamman R. ASE statement on point-of-care ultrasound during the 2019 novel coronavirus pandemic. J Am Soc Echocardiogr. 2020;33(6):670-3.

14. Huang G, Vengerovsky A, Morris A, Town J, Carlbom D, Kwon Y. Development of a COVID-19 point-of-care ultrasound protocol. J Am Soc Echocardiogr. 2020;33(7):903-5. https://doi.org/10. 1016/j.echo.2020.04.023. Epub 2020 Apr 23 PMID: 32624091.

15. Khanji MY, Ricci F, Patel RS, Chahal AA, Bhattacharyya S, Galusko V, et al. Special Article - The role of hand-held ultrasound for cardiopulmonary assessment during a pandemic. Prog Cardiovasc Dis. 2020;63(5):690-695. https://doi.org/10. 1016/j.pcad.2020.07.003. Epub 2020 Jul 10. PMID: 32659342; PMCID: PMC7351031.11.

Publisher's Note Springer Nature remains neutral with regard to jurisdictional claims in published maps and institutional affiliations. 